LBNL-56075

\title{
A First-Generation Prototype Dynamic Residential Window
}

\author{
Christian Kohler, Howdy Goudey, and Dariush Arasteh \\ Windows and Daylighting Group \\ Lawrence Berkeley National Laboratory \\ Berkeley CA 94720
}

October 26, 2004

\begin{abstract}
We present the concept for a "smart" highly efficient dynamic window that maximizes solar heat gain during the heating season and minimizes solar heat gain during the cooling season in residential buildings. We describe a prototype dynamic window that relies on an internal shade, which deploys automatically in response to solar radiation and temperature. This prototype was built at Lawrence Berkeley National Laboratory from commercially available "off-the-shelf" components. It is a stand-alone, standard-size product, so it can be easily installed in place of standard window products. Our design shows promise for near-term commercialization. Improving thermal performance of this prototype by incorporating commercially available highly efficient glazing technologies could result in the first window that could be suitable for use in zero-energy homes. The unit's predictable deployment of shading could help capture energy savings that are not possible with manual shading. Installation of dynamically shaded windows in the field will allow researchers to better quantify the energy effects of shades, which could lead to increased efficiency in the sizing of heating, ventilation, and air conditioning equipment for residences.
\end{abstract}

\section{Background}

Although the introduction of low-emissivity (low-E) glazing and other improvements in window technology have significantly reduced window-related energy use and peak demand in residential buildings during the past 15 years, today's most efficient windows are still not efficient enough for zero-energy buildings (ZEBs) - buildings that consume no net or non-renewable energy (Apte et al. 2003). For ZEBs to become a reality, windows will have to shift from being a source of net energy loss to a source of net energy gain. One approach to modifying windows so that some energy gain is possible is to regulate the solar heat gain through them. In heating-dominated and mixed climates, solar gains are beneficial in winter, but they increase cooling load and peak demand in summer. For a window to provide net energy gains in these climates, it should have a low 
U-factor ${ }^{1}$ and high solar heat gain when the building needs heating. In coolingdominated climates, the primary concern is to keep solar gains through windows as low as possible in summer.

\section{Dynamic Window Design Concept}

To increase window efficiency to meet the performance requirements of ZEBs and to optimize winter solar gains, we have investigated window designs incorporating dynamic solar heat gain properties that sense and respond to daily and seasonal changes in climate conditions. Dynamic windows' low solar gains in summer can minimize overall and peak cooling demand, and high solar gains combined with insulation properties in winter can maximize solar heating effects, saving significant energy year round in residences in most climates.

Dynamic residential windows could be based on a number of possible technologies, including built-in shades, electrochromic coatings, or reflective metal hydride coatings. A dynamic window with an automatically deployable shade, for example, might operate as follows: on days when the house needs heating, the shade would be open as much as possible, admitting maximum solar gain; on days when the house needs cooling, the shade would be closed as much as possible, minimizing solar gain. During the night, the shade could also be deployed to achieve a lower U-factor. Based on this principle, future dynamic windows constructed with highly efficient components could actually be net energy gainers in residential buildings in much of the United States (see Figure 1).

One goal of our research is to develop a stand-alone, self-contained dynamic window unit that can be installed in place of existing window products already on the market and that is not significantly more expensive than current highly efficient windows. We want to develop a dynamic window unit that does not require specialized preparation of the building envelope or specialized knowledge on the part of the installer. The window should be the same size as other standard window products and should provide its own electric power and control algorithm so that it can operate independently, without specialized wiring or power sources, though it should also have the potential to be connected in a network with other windows in a residence.

\section{The Prototype}

Because one of our goals is to keep the price of our dynamic window reasonable, we chose to create a prototype that relies on a built-in deployable shade. This prototype can be created for moderate cost from readily available, "off-the-shelf” components.

The prototype that we constructed has multiple glazing layers and a built-in shade that deploys automatically in response to sunlight and temperature (see Figure 2).

\footnotetext{
${ }^{1} \mathrm{U}$-factor is a measure of the rate of heat flow through a window and is the inverse of the R-value, which is a measure of the window's insulation value (thermal resistance). The lower the U-factor, the greater a window's resistance to heat flow (i.e., loss of heat from a building's interior). U-factors are expressed in $\mathrm{Btu} /\left(\mathrm{h}-\mathrm{ft}^{2}-\mathrm{F}\right)$.
} 


\section{Glazing and Shade}

The prototype was constructed using a commercially available, three-layer, aluminumclad wood window that contains a single pleated fabric shade between exterior double glazing and interior single glazing (see Figure 3). The exterior double glazing encloses argon gas fill. The cavity side of the double glazing has a spectrally selective low-E coating, which was standard on the ready-made window that we used for our prototype but would not be appropriate for the final design because the spectrally selective coating limits the amount of solar heat gain through the window when the shade is open, thereby limiting the window's effectiveness for offsetting heating loads during the winter. The single glazing on the room side of the window unit has a durable high solar gain low-E coating (on the surface that faces the built-in shade). The single glazing is removable so that the shade can be accessed in case of malfunction, etc. The shade (without motor) is a standard product upgrade.

\section{Controls}

To the standard window with built-in shade, we added computerized controls, sensors, and a 12-volt direct current (DC) motor to drive the shade. The computer electronics (a circuit board and chip and a real-time, Java-based microcontroller), sensors (including a digital thermometer and solar detector), and motor are embedded in the window headrail (see Figure 4).

The computer employs a control algorithm that raises and lowers the shade in response to sunlight and temperature after the building occupant initially activates the shade for the day. For example, once the shade is opened manually by the occupant in the morning, it is raised and lowered automatically during the day in response to increasing and decreasing temperatures and solar radiation levels; in the evening, the shade is lowered in response to darkness (see Figure 5). This schedule could be customized, and a button allows the occupant to override the product's current setting and raise or lower the shade at will. (A time-series video of the window in operation can be viewed at http://windows.lbl.gov/projects/dynamicshades).

An example of a typical operation cycle in response to changing weather conditions during one day is as follows:

- In the morning, the shade is down; the occupant pushes the override button, and the shade rises.

- At mid-morning, direct solar radiation strikes the window, and outdoor temperatures are warmer than 78 degrees Fahrenheit (F); the shade automatically lowers.

- In the afternoon, the temperature falls below 74 degrees $\mathrm{F}$; the shade rises automatically (because solar gain through the window is unlikely to add to the interior cooling load under these weather conditions).

- In the evening, darkness falls, and the shade automatically drops to provide privacy and nighttime insulation.

At any time, the override button can be pushed to change the shade's position.

The inputs to the prototype's controls are simple: exterior temperature, solar intensity, and the override button. Although the algorithm does not currently take occupant 
behavior into account, an occupancy sensor could be added (e.g., so that, for example, if the occupant manually raised the shade on a hot day when the house requires cooling, the shade would lower automatically once the occupant left the room). The unit could also be programmed so that the shade would be raised automatically after a certain hour (e.g., noon) if the occupant had not already opened the shade (on days when the house requires heating and solar gain would be an advantage). Other programming options could be created to address window operation when occupants are on vacation, etc.

The shade motor on the prototype draws two Watt hours (Wh) per month if the shade is raised and lowered six times during the day. The prototype is currently powered by an external 12-volt source, but the limited number of shade cycles expected per day makes it practical for the window to rely on a photovoltaic (PV) power source and batteries. Improvements will be required if PV power is to be used, however, because the control electronics currently require a continuous supply of more power $(1,200 \mathrm{Wh} / \mathrm{month})$ than a PV power source can support. Solutions for future models include incorporating a "sleep" mode for the control system and using new low-power nodes such as those based on the emerging Zigbee standard for wireless sensors and actuators that are designed for low-power battery operation and claim to draw less than $1 \mathrm{Wh} / \mathrm{month}$, or other small, simple, programmable microcontrollers that consume $1.5 \mathrm{Wh} /$ month.

We performed some preliminary calculations of the energy that would be available if PV cells were attached to the window. Assuming 0.5 square feet $\left(\mathrm{ft}^{2}\right)$ of $\mathrm{PV}$ and four Watts actual capacity, we calculate that the PV cells would produce $24 \mathrm{Wh} /$ month on northfacing windows and $104 \mathrm{Wh} /$ month windows on all other orientations. These calculations were based on the National Renewable Energy Laboratory PV-Watts program and used climate data for the worst (i.e., least sunny) month in Minneapolis MN, Phoenix AZ, and Baltimore MD (which represent a range of climates).

PV-generated power would be stored in batteries within the window. Although some power losses are associated with charging and discharging, the PV-battery system would provide sufficient energy as long as the controller draws no more than $10 \mathrm{Wh} /$ month.

\section{Thermal Performance}

The additional insulation provided by the lowered shade at night means that the prototype's nighttime performance exceeds that of a product with triple glazing. Because the shade deploys automatically at night, the unit's energy performance during nighttime hours will also exceed that of windows whose shades are not normally drawn by occupants at night. The window's increase in thermal performance results not only from the reliable deployment of the shade but also from the shade's location between the glazing, which gives it better insulating properties than a shade mounted on the room side of a window's surface. The replacement of the outer double-glazed, low-E-insulated glazing unit in our prototype with more highly insulating glazing (e.g., three layers, one of which is a low-E suspended film) could further improve the unit's thermal performance, as would placement of the shade as close to the window's exterior (outdoor-side) glazing as possible. (The placement of the shade between exterior double 
and interior single glazing in our prototype is a result of our having chosen to use an "offthe-shelf" window product).

Commercialization and Energy-Efficiency Potential

The "smart" design of our prototype could be adopted by manufacturers in the near term, and its general design could be readily applied to a product with electrochromic (EC) glazing, which appears to be a promising technology for the long term (research on EC materials is not part of the current project).

Field installation of dynamically shaded windows would enable energy researchers to better quantify the impact of predictably deployed shades on residential energy use and heating, ventilation, and air-conditioning (HVAC) equipment sizing. Manually deployed shades are not typically considered to have a significant impact on HVAC equipment sizing. However, because shades in a dynamic window are deployed in a predictable manner, they are expected to capture energy savings missed by manually controlled shades. Figure 1 shows the potential energy savings for a dynamically shaded high solar gain triple glazed window ("Prototype Dynamic") compared with other window technologies. (Calculations assume a high solar gain window, which enables capturing more of the solar gains to offset heating, unlike our prototype which used the stock low solar gain low-e.) We expect that a dynamically shaded window would have a U-factor better than 0.25 (Btu/ $\left.\mathrm{ft}^{2}-\mathrm{hr}-\mathrm{F}\right)$, which is the rated value for the off-the-shelf window product we used to construct our prototype. The solar heat gain coefficient is estimated at 0.15 when the shade is drawn (this will vary with shade type and color). Some modifications to our prototype design would improve thermal performance, including relocating the shade to be nearer the window's outdoor surface. This modification should improve the window's performance sufficiently that it could be used effectively in ZEBs in mixed and heating-dominated climates. In ZEBs in cooling-dominated climates, dynamic shading should be combined with a low solar heat gain glass and building shading elements.

\section{Next Steps}

Having completed and demonstrated the initial prototype of a dynamically shaded window, we envision future research and improvements in the areas of new component technologies and quantification of energy-savings potentials. Some key issues for future work include:

- incorporating PV power supply as described above, contained within the window unit

- developing capability to network dynamic windows so that each window in a group installation would not require a full complement of electronics; similarly, researching use of a sensor package for all windows in a house rather than placing individual sensors on each window

- developing the capability to integrate dynamic window operation with "smart" thermostat operation and real-time energy pricing

- improving control algorithms to incorporate options described above (e.g., vacation schedules) 
- incorporating motion/occupancy sensors (so that, for example, an override of shade position would automatically "expire" after sensor does not detect motion in the room for a predetermined period of time)

- exploring use of ventilation sensor(s) for operable window design, which could open/close windows in response to changes in relative humidity, indoor air quality, and heating/cooling conditions

\section{Conclusions}

Our dynamically shaded window prototype shows promise for significant year-round energy savings in mixed and heating climates. Summer energy savings could also result in cooling-dominated climates from dynamic shading if combined with fixed, low solar heat gain glass. Our prototype design could be commercialized in the near term once it is improved as described above, optimized for manufacturing and its reliability can be demonstrated to meet industry requirements. From the future steps noted in the section above, key improvements to be undertaken in the immediate future include: optimizing the power source, minimizing power consumption by the control electronics, improving sensors and communications, and demonstrating the reliable performance of the window and its occupant interaction features in the field.

\section{References}

Apte, Joshua; Dariush Arasteh;Yu Joe Huang. 2003. "Future Advanced Windows for Zero-Energy Homes.” ASHRAE Transactions, v. 109, pt. 2.

Lawrence Berkeley National Laboratory. Dynamic Shades project. http://windows.lbl.gov/projects/dynamic shades.

National Renewable Energy Laboratory. PV Watts program. http://rredc.nrel.gov/solar/calculators/PVWATTS/

\section{Acknowledgments}

This work was supported by the Assistant Secretary for Energy Efficiency and Renewable Energy, Office of Building Technology, State and Community Programs, Office of Building Systems of the U.S. Department of Energy under Contract No. DE-AC03-76SF00098.

The prototype window is a customized version of a commercially available product provided to us by the Pella Corporation.

The authors would like to acknowledge Nan Wishner for her invaluable assistance in editing this paper and to Luís Fernandes for his help with illustrations. 


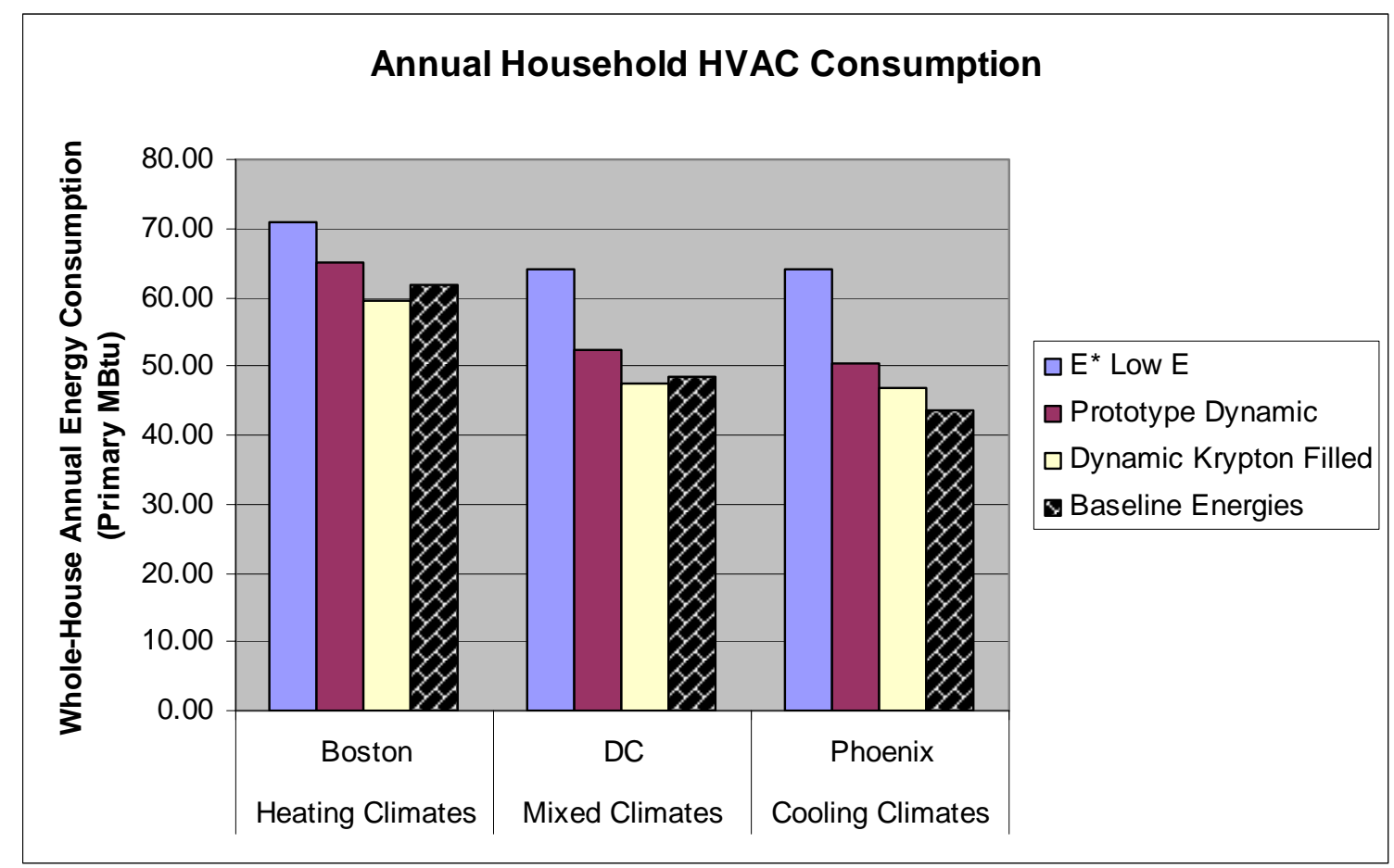

Figure 1: Energy Consumption of various window technologies

\begin{tabular}{|c|c|c|}
\hline Window & $\begin{array}{c}\text { U-factor } \\
\left(\mathrm{Btu} / \mathrm{ft}^{2}-\mathrm{hr}-\mathrm{F}\right) / \\
\left(\mathrm{W} / \mathrm{m}^{2}-C\right)\end{array}$ & SHGC \\
\hline Baseline Loads (no windows) & $\mathrm{N} / \mathrm{A}$ & $\mathrm{N} / \mathrm{A}$ \\
\hline $\begin{array}{l}\text { Low-E; (current ENERGY STAR }{ }^{\mathrm{TM}} \text { ) } \\
\text { Phoenix } \\
\text { DC } \\
\text { Boston }\end{array}$ & $\begin{array}{l}0.40(2.27) \\
0.40(2.27) \\
0.35(1.98)\end{array}$ & $\begin{array}{l}0.30 \\
0.55 \\
0.60\end{array}$ \\
\hline $\begin{array}{l}\text { Prototype Triple glazing with dynamic } \\
\text { shade }\end{array}$ & $\begin{array}{l}0.23 \text { (1.31) (with } \\
\text { shade deployed) } \\
0.25 \text { (1.42) (with } \\
\text { shade open) }\end{array}$ & $\begin{array}{l}0.15 \\
0.46\end{array}$ \\
\hline $\begin{array}{l}\text { Dynamic Triple glazing with multiple } \\
\text { low-E and krypton fill }\end{array}$ & $0.10(0.57)$ & $\begin{array}{l}0.10 \text { (with shade } \\
\text { deployed) } \\
0.35 \text { (with shade } \\
\text { open) }\end{array}$ \\
\hline
\end{tabular}

Data in table is based on Apte, Arasteh, and Huang 2003.) 


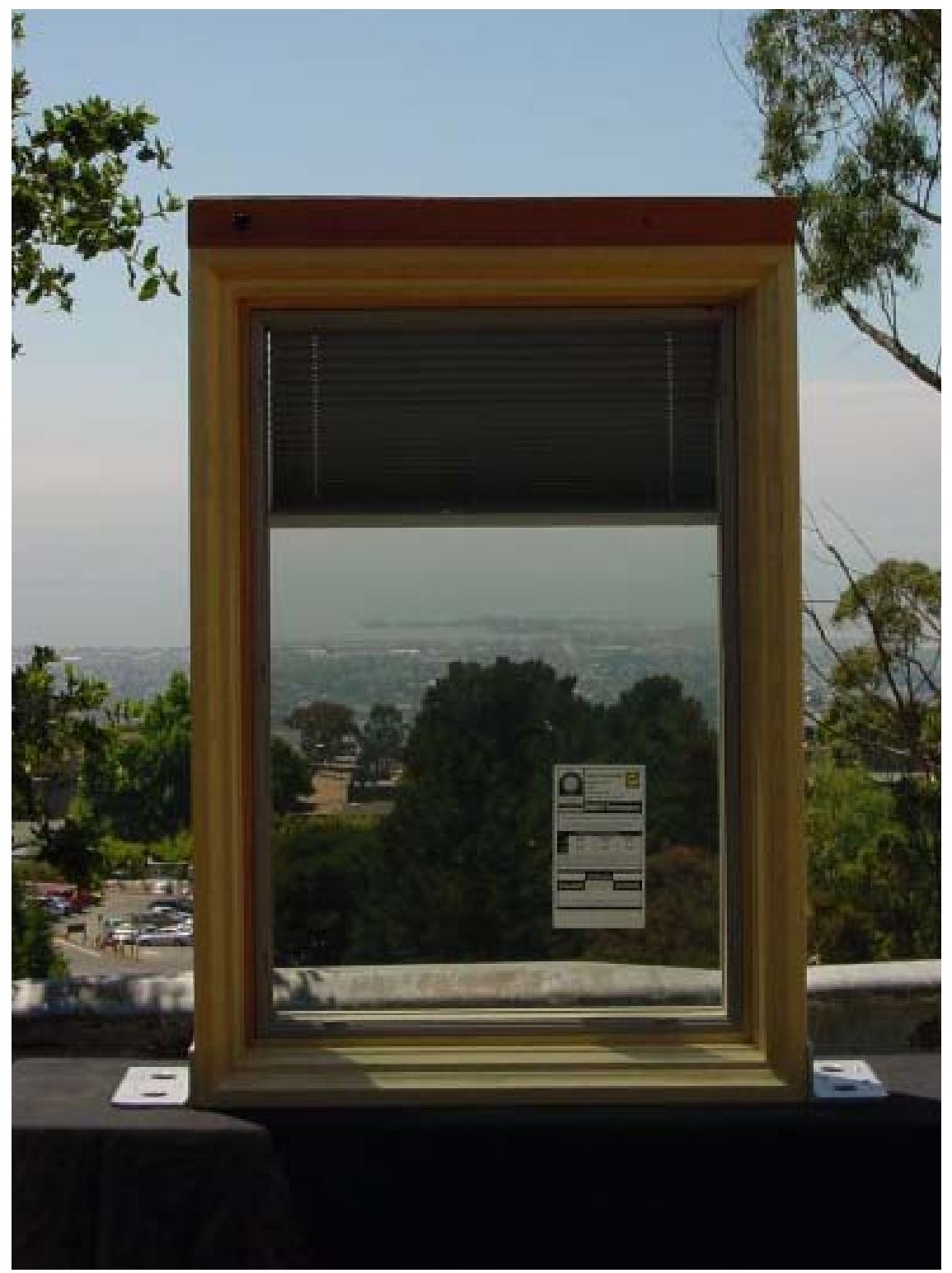

Figure 2: Prototype Window (Shades in the process of closing to respond to direct solar radiation) 


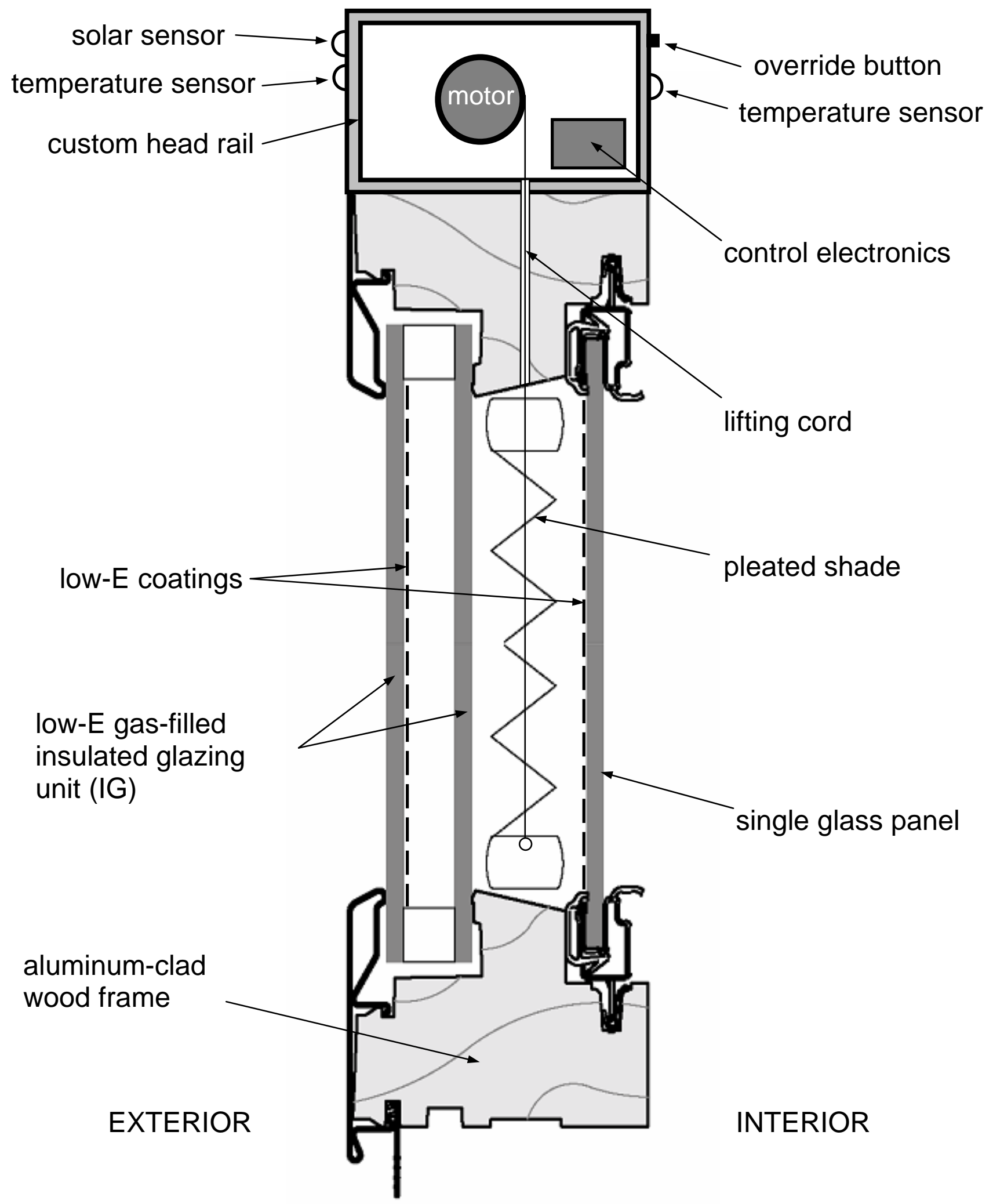

Figure 3: Schematic of Dynamic Window 


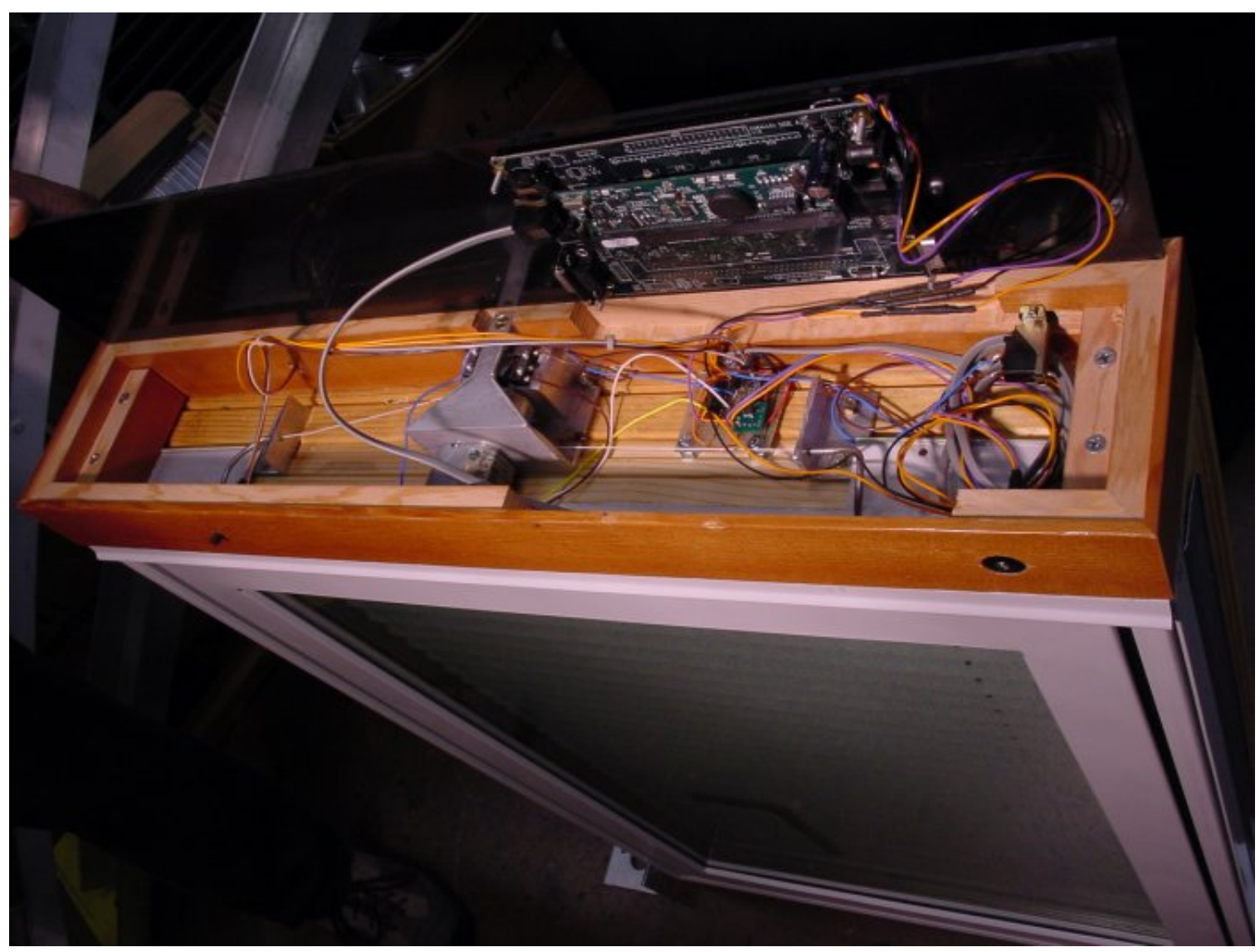

Figure 4: Headrail of Dynamic Window showing controller and sensors 


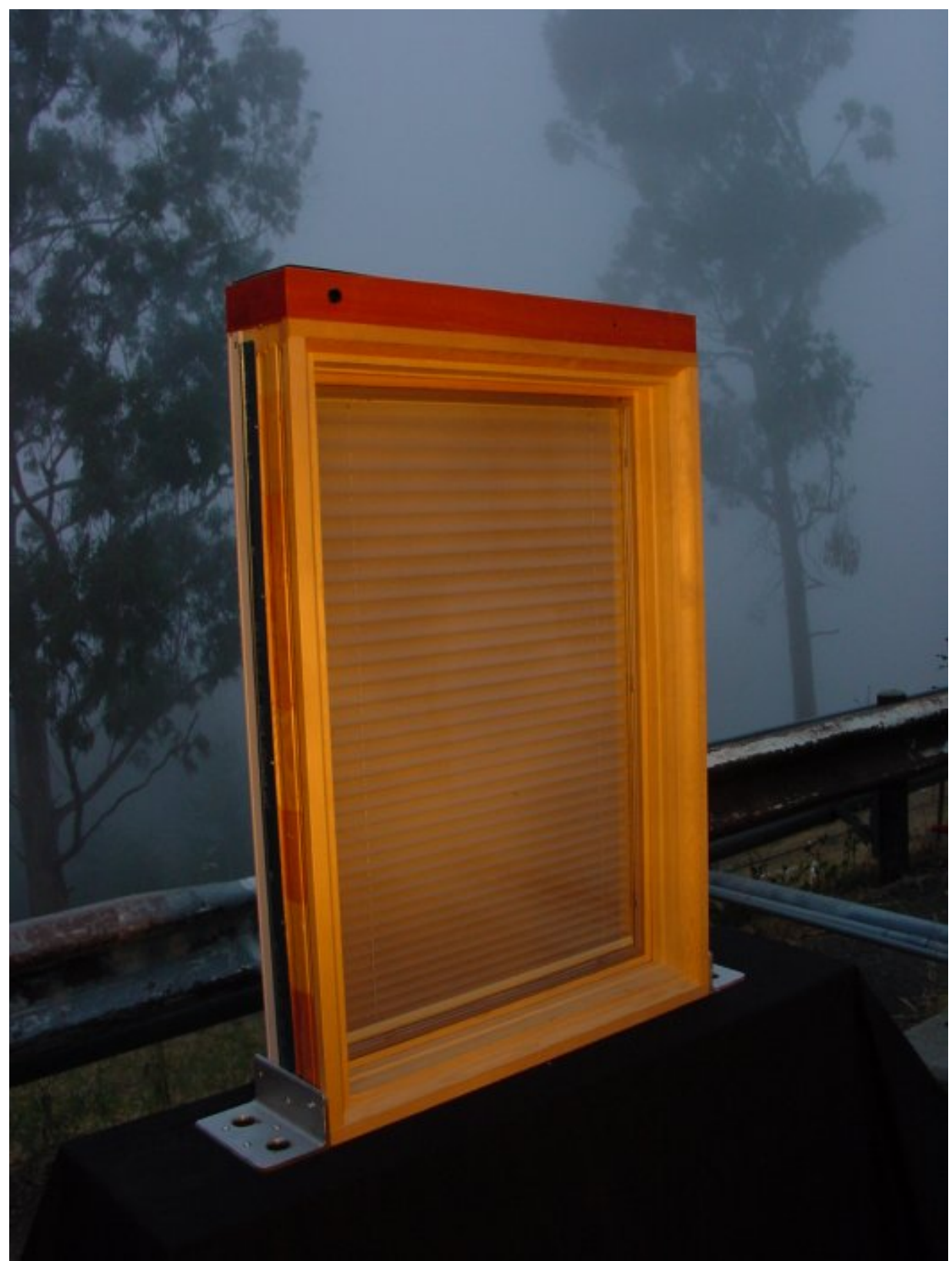

Figure 5: Prototype Dynamic Window at Dusk (Shades closed for privacy and increased insulation) 